\title{
Comparative Study of Deposit through a Membrane and Spin-Coated MWCNT as a Flexible Anode for Optoelectronic Applications
}

\author{
Walid Aloui, Saidi Hamza, and Abdelaziz Bouazizi \\ Laboratory of Physics of Condensed Matter and Nanosciences, Faculty of Sciences of Monastir, Avenue of the Environment, \\ 5019 Monastir, Tunisia \\ Correspondence should be addressed to Walid Aloui; alouiwalid26@yahoo.fr
}

Received 20 December 2015; Accepted 7 February 2016

Academic Editor: Hassan Karimi-Maleh

Copyright (C) 2016 Walid Aloui et al. This is an open access article distributed under the Creative Commons Attribution License, which permits unrestricted use, distribution, and reproduction in any medium, provided the original work is properly cited.

We present a comparative study between multiwalled carbon nanotubes (MWCNTs) thin films deposited on polyethylene terephthalate (PET) substrates using (i) spin-coating technique and (ii) deposition through a membrane. We deduce from transparence, electrical properties, and AFM image that deposition through membrane presents better properties than spincoating method. The concentration comparison shows that the optimum result was achieved at a concentration of $1.2 \mathrm{mg} \cdot \mathrm{mL}^{-1}$ corresponding to a resistance $\left(R_{s}\right)$ of $180 \Omega \cdot \mathrm{cm}^{-2}$ and an optical transparence of about $81 \%$ using a wavelength $550 \mathrm{~nm}$. We will also demonstrate the use of the elaborated electrodes to fabricate the following flexible structure: PET-MWCNTs/MEH-PPV/Al. The series resistance $R_{s}$ and the ideality factor $n$ were calculated.

\section{Introduction}

Actually, transparent conductive films are extremely common and critically important in optoelectronic devices. They are used as electrodes for E-readers and digital cameras [1], photovoltaic devices such as solar cells [2], and organic lightemitting diodes (OLEDs) [3]. Currently, tin doped indium oxide (ITO) presents the dominant transparent conductive material with growing annual demand at 20\% [4]. ITO has been studied and refined for over 70 years. As a result, the material offers many beneficial properties. However, ITO present certain inconveniences, mainly reflected on the depleted supply of raw materials and their brittleness. The supply of indium is constrained by both mining and geopolitical issues, which leads to dramatic price fluctuations over the last decades. The high price of indium determined the high cost of ITO, since they compose nearly $75 \mathrm{wt} \%$ of a typical ITO film [5]. The current devices are typically based on rigid substrates. As ITO tends to fracture at strains of $2 \%$, it is completely unsuitable for using in flexible electronics. Therefore, new transparent electrode materials have rapidly emerged in recent years, including carbon nanotubes (CNTs), graphene, and metal nanowires. This material presents good conductivity coupled with aspect ratio which yields films with high transparence, adequate sheet resistance, and high mechanical flexibility [6, 7]. The intrinsically good conductivity coupled with aspect ratio yields films with high transparence, adequate sheet resistance, and high mechanical flexibility. These material properties, combined with the low costs, deposition, and the inexpensive material, make these emerging nanomaterials very attractive. Among the dominant nanoscale materials, CNTs are the most promising and mature materials intensively investigated. Indeed, CNTs exist in two types: the single-walled carbon nanotubes (SWCNTs) and the multiwalled carbon nanotubes (MWCNTs). Much research was devoted to the application of SWCNTs to optoelectronics for their good properties [69]. On the other hand, MWCNTs are easier to process than SWCNTs; MWCNTs are also less liable to form tight clusters and are comparatively cheaper. Also, MWCNTs are even more suitable for the implementation of charge transport and charge transfer, because of the metallic conductivity and the predictable HOMO-LUMO energy levels [10]. They are employed as hole-injecting electrodes or charge transport 


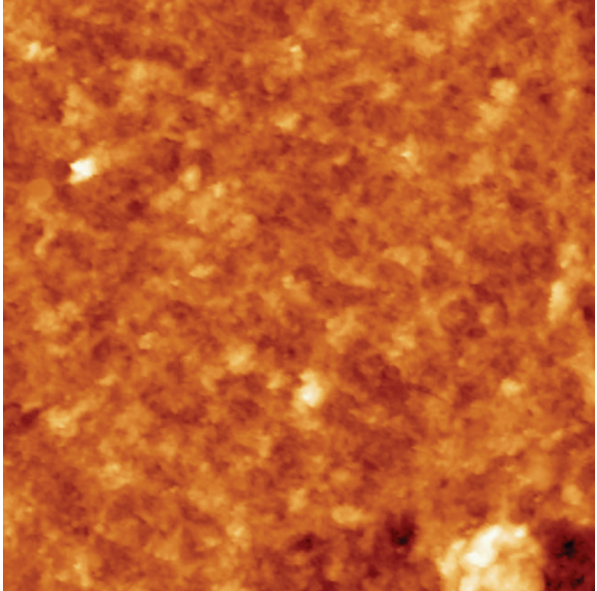

(a)

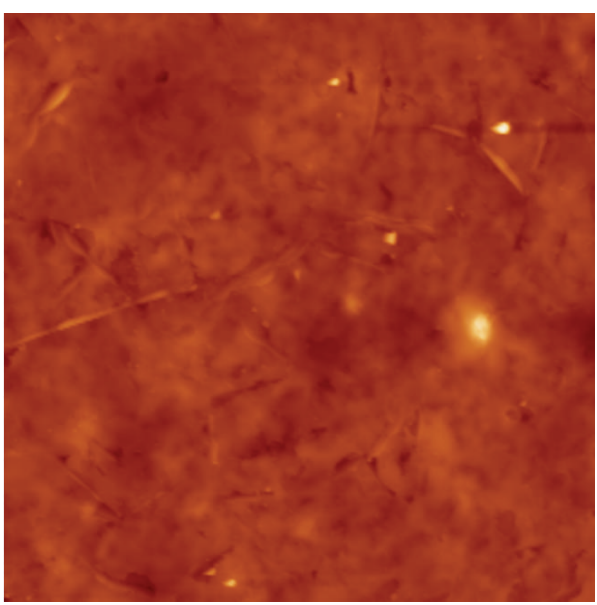

(c)

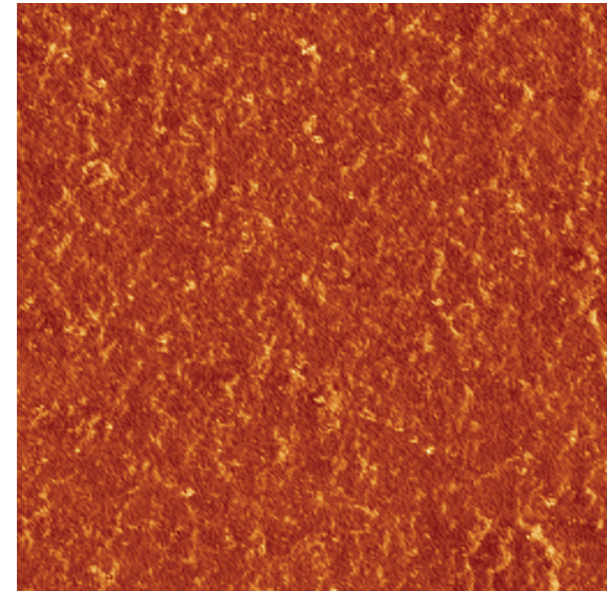

(b)

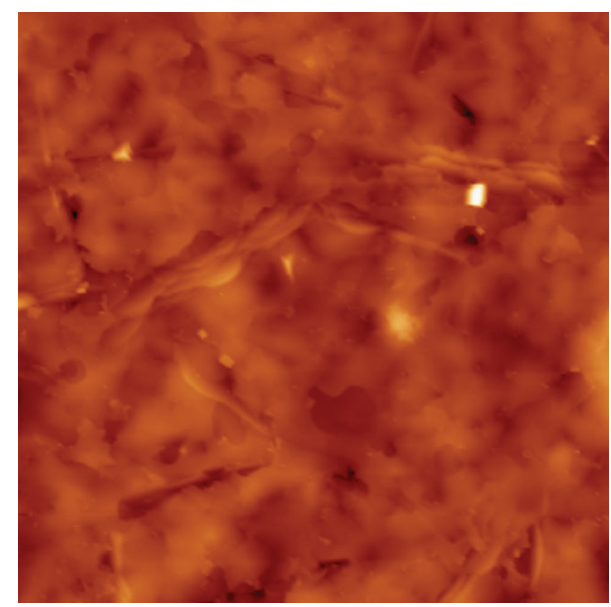

(d)

Figure 1: AFM image of CNTs on the PET substrate: deposited by spin coating at concentrations of (a) $1.2 \mathrm{mg} \cdot \mathrm{mL}^{-1}$ and (b) $2.5 \mathrm{mg} \cdot \mathrm{mL}^{-1}$; deposited through a membrane at concentrations of (c) $1.2 \mathrm{mg} \cdot \mathrm{mL}^{-1}$ and (d) $2.5 \mathrm{mg} \cdot \mathrm{mL}^{-1}$.

layers [11-16]. For those reasons, we will use MWCNTs as flexible electrodes. We will compare two types of flexible electrodes based on MWCNTs, elaborated by spin coating and through a membrane. Then, we will demonstrate their use as transparent electrodes for the following structure: PETMWCNTs/MEH-PPV/Al.

\section{Experimental Details}

MWCNTs were dispersed in deionized water containing $1 \mathrm{wt} \%$ sodium dodecyl sulfate (SDS). Then, MWCNTs coated electrodes were prepared using two methods in order to create uniform networks of MWCNTs on flexible substrates. Briefly, MWCNTs were vacuum-filtered on mixed cellulose ester membranes (MCE) and then transferred onto PET substrates (described in our previous work) [16]. MEH-PPV polymer was dispersed in THF solvent at a concentration of $15 \mathrm{mg} \cdot \mathrm{mL}^{-1}$ and then spin-coated on the PET-MWCNTs at an angular speed of $1500 \mathrm{rpm}$ for $10 \mathrm{~s}$. Finally, aluminum top electrodes were deposited by thermal evaporation through a shadow mask.

\section{Results and Discussion}

3.1. Morphological Study by AFM. In order to explore the topography of the CNTs, AFM studies should be performed. We present in Figure 1 the AFM images of CNTs anode deposited by spin coating at concentrations of (a) $1.2 \mathrm{mg} \cdot \mathrm{mL}^{-1}$ and (b) $2.5 \mathrm{mg} \cdot \mathrm{mL}^{-1}$ and through a membrane adopting the same concentrations ( $c$ and d, resp.). The AFM images of spin-coated film show that CNTs are condensed on themselves without any specified direction. In addition, we observe that aggregates domains are formed (Figure 1(b)). This result is due to the Van Der Waals forces among the tubes and their hydrophobicity. We note that $\pi-\pi^{*}$ interaction between different tubes is the origin of the aggregations observed [17]. This is expected due to the deposition method used to put the CNTs on PET substrate.

Using the second method, CNTs become clearly observed. AFM images exhibit that CNTs networks are more densified and characterized by a network of cylindricallike features, and the approximate length is about several hundreds of nanometers and the width is much smaller. 


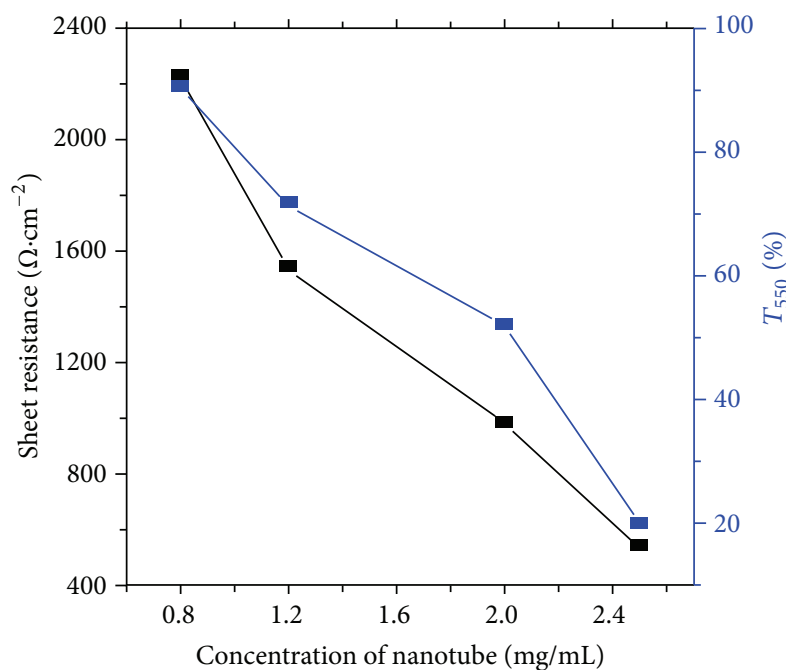

(a)

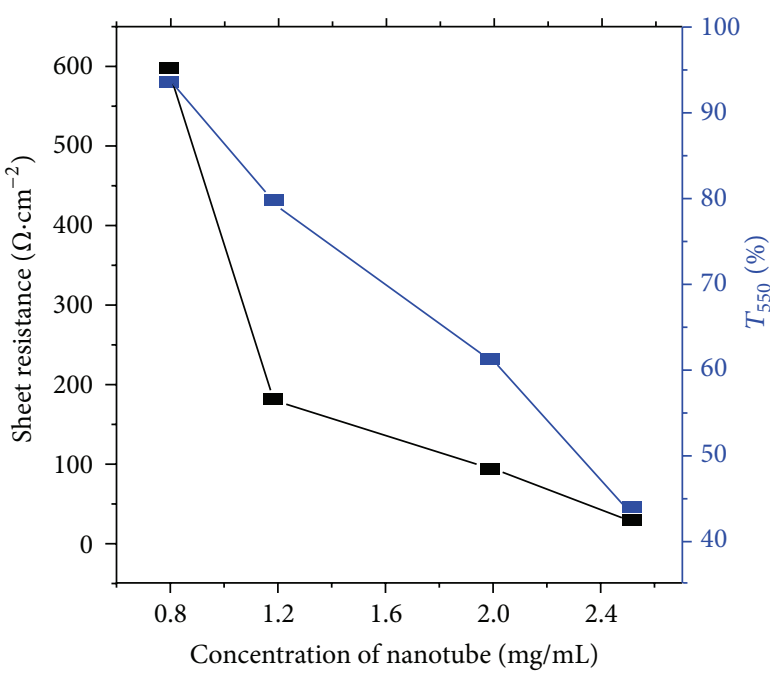

(b)

FiguRE 2: Variation of resistance $R_{s}$ and transmission $T$ for different concentrations. (a) Anode elaborated by spin coating; (b) anode elaborated through a membrane.

These well-organized overlapping structures may result from the capillary force and the force induced by the evaporation; it is like the photonic crystals formation mechanism. This alignment can lead to better performance for optoelectronics devices [18]. In addition, the space area unoccupied by CNTs is reduced. This leads to obtaining a solid conductive film and a good optical transparency level. Indeed, for the concentration of $1.2 \mathrm{mg} \cdot \mathrm{mL}^{-1}$, interconnected CNTs looked like a spider network. This could create a rapid charge transport between the interconnecting conductive paths of MWCNTs and a high optical transparency level due to well-developed structures [19].

3.2. Electrical and Optical Properties. An encouraging optical properties' result, comparable to that obtained for the ITO, was found with $0.8 \mathrm{mg} \cdot \mathrm{mL}^{-1}$ and $1.2 \mathrm{mg} \cdot \mathrm{mL}^{-1}$. However, the CNTs network retains high transparency to the visible and near infrared range of the electromagnetic spectrum. Obviously, we found that CNTs transmission is mainly due to the absorbance inside film [20]. Indeed, for the other two concentrations, transparency measurement shows that these films are opaque. This interpretation is correlated with AFM morphology. Accordingly, these structures can be good candidates to be integrated as anodes in photovoltaic devices. The variation of the surface resistance $R_{s}$ and the transmission $T$ are shown in Figure 2, for MWCNTs anode deposited by spin coating. Obviously, it is important to note that the distribution of MWCNTs plays a crucial role for optical transparency measurements and for networks electrical conductivity.

Indeed, the increase of MWCNTs concentration leads to the decrease of $R_{s}$; thicker layers (low transmission) exhibit better electrical properties due to the increase of the percolation path number so that electrons can move through. However, for the concentration $0.8 \mathrm{mg} \cdot \mathrm{mL}^{-1}$, PETMWCNTs films exhibit a high resistance in the order of
$2.21 \mathrm{~K} \Omega \cdot \mathrm{cm}^{-2}$ and an optical transmission of $91 \%$ at $550 \mathrm{~nm}$. For the concentration $2.5 \mathrm{mg} \cdot \mathrm{mL}^{-1}, R_{s}$ decreased significantly to $580 \Omega \cdot \mathrm{cm}^{-2}$ with an optical transmission of $20 \%$. The resistance decrease was effective because of charge transport percolation paths through the well-interconnected networks [21]. The $R_{s}$ resistance is high compared to the result obtained with the method using an ITO substrate. Consequently, we cannot use them as transparent electrodes in optoelectronic devices.

Transparency and conductivity variations of flexible anode elaborated through a membrane are shown in Figure 2. For the concentration $2.5 \mathrm{mg} \cdot \mathrm{mL}^{-1}$, PET-MWCNTs films present a high resistance in the order of $587 \Omega . \mathrm{cm}^{-2}$ and an optical transmission of $43 \%$. For the concentration $1.2 \mathrm{mg} \cdot \mathrm{mL}^{-1}$, sheet resistance decreased significantly to $180 \Omega \cdot \mathrm{cm}^{-2}$ with an optical transmission approximately $81 \%$ at $550 \mathrm{~nm}$. This can satisfy the requirement for the application in touch screen [22] and is comparable to most of the literature results [23]. Obviously, it is noted that the transmission and the surface resistance depend mainly on the distribution of MWCNTs on the substrate. Indeed, it is clear that increase of MWCNTs concentration leads to the decrease of $R_{s}$. This can be attributed to three major factors [24, 25]: (i) the intrinsic conductivity of MWCNTs, (ii) contact resistance tube-tube, and (iii) the concentration and the distribution of MWCNTs on the PET substrate.

The SDS covering the MWCNTs surface increases the contact resistance. Hence, it is required to remove SDS residues after films elaboration. Cui et al and Wang et al. $[26,27]$ studied the effect of the removal of residual SDS from CNTs network and they showed that the conductivity increase is mainly due to the effective removal of residual SDS and not from a chemical doping effect. In contrast, we made a similar experience with our film. Indeed, we washed the films with chlorobenzene, acetone, and pure water. Thus, we note an improvement in conductivity due to 


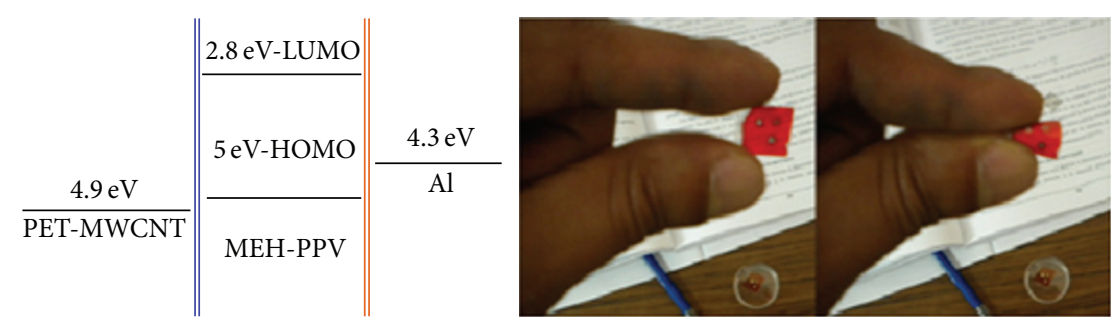

FIGURE 3: Energy diagram and picture of the flexible structure PET-MWCNTs/MEH-PPV/Al elaborated.

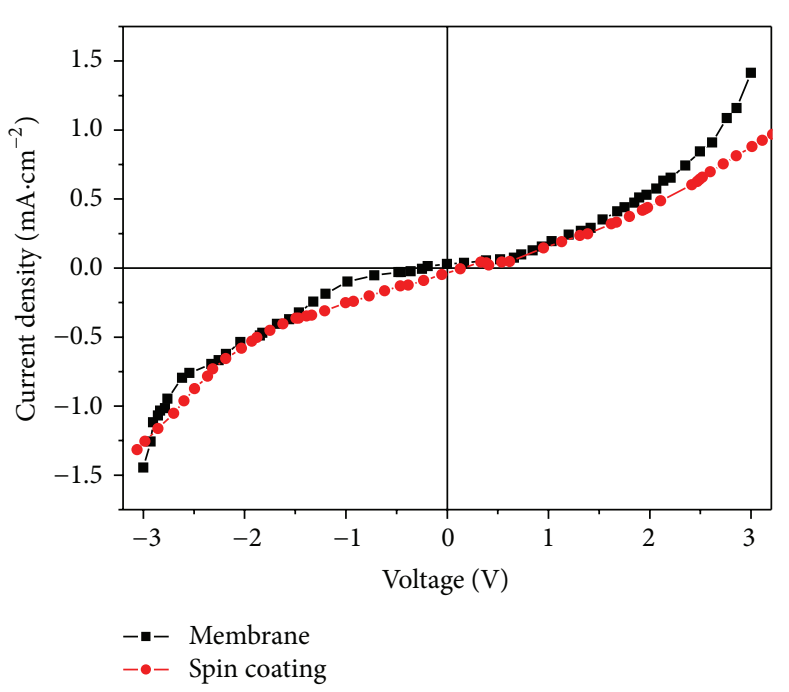

(a)

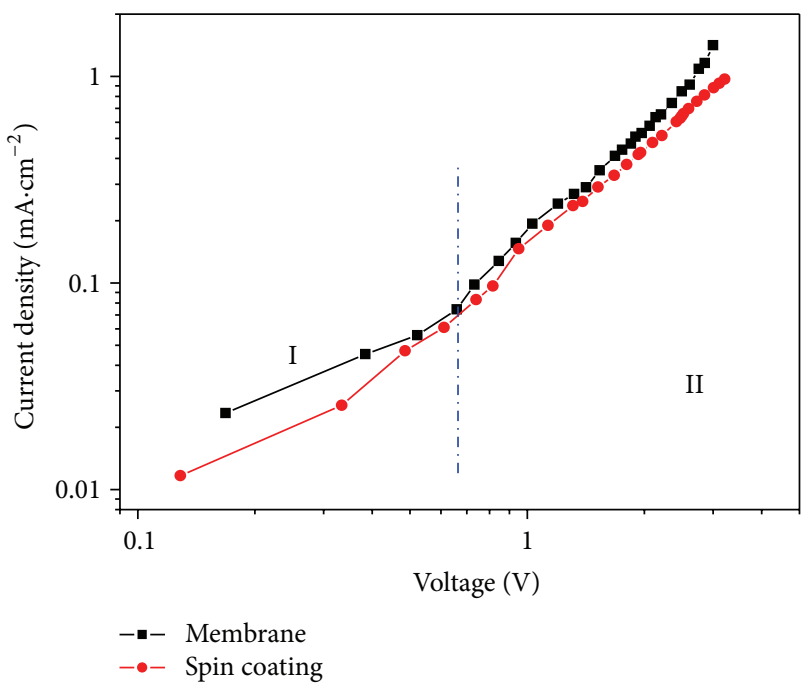

(b)

FIgURE 4: $J-V$ characteristic for PET-MWCNTs/MEH-PPV/Al structure in (a) linear scale and (b) logarithmic scale.

the better connection between the MWCNTs. Our results are comparable to other similar works reported in the literature. Ko et al. [21] and Castro and Schmidt [28] found an $R_{s}$ resistance of $370 \Omega \cdot \mathrm{cm}^{-2}$ with a transmission $T_{550}$ of $77 \%$ and of $20 \mathrm{~K} \Omega \cdot \mathrm{cm}^{-2}$ with a transmission $T_{550}$ of $78 \%$, respectively.

\subsection{Electrical Properties of \\ PET-MWCNTs/MEH-PPV/Al Structures}

3.3.1. Current Density-Voltage Characteristic. In order to demonstrate the efficiency of our flexible electrodes, we elaborate the following structure: PET-MWCNTs/MEH-PPV/Al. Figure 3 shows their energy diagram with a picture of the elaborated structure.

Current density-voltage characteristic of PET-MWCNTs/ $\mathrm{MEH}-\mathrm{PPV} / \mathrm{Al}$ is shown in Figure 4. The applied voltage during the measurement varies from -3 to $+3 \mathrm{~V}$. The positive polarity corresponds to side of the PET-MWCNTs electrode. Consider the output work values of the MWCNTs and aluminum, which are, respectively, about $4.9 \mathrm{eV}$ and $4.3 \mathrm{eV}$ and from the gap energy of the MEH-PPV conjugated polymer $\left(E_{\mathrm{HOMO}}=5 \mathrm{eV}\right.$ and $\left.E_{\mathrm{LUMO}}=2.8 \mathrm{eV}\right)$. We conclude that the holes injection barrier $(\sim 0.1 \mathrm{eV})$ in the MEH-PPV polymer is localized at the MWCNTs/MEH-PPV interface. This barrier height allows charge injection by thermoionic emission. An Ohmic behavior is obtained for the structure elaborated on the MWCNTs using spin coating. These results could be explained by the low thickness of the active layer which allows direct connections (short circuit) between the cathode and the anode. However, we always find the same Ohmic behavior when using other film thicknesses obtained with different deposition rates. This can also be related to the MWCNTs orientations, which present a several micrometers of length. On the other hand, for the structure prepared on the MWCNTs elaborated through membrane, the curve is nonlinear and it has a rectifier behavior due to the injection charge carriers from MWCNTs electrode to the active layer. We obtain an organic diode behavior with a threshold voltage $V_{s}$ close to $1.9 \mathrm{~V}$. Our results are comparable to many other published studies [29].

The conduction mechanisms that control OLEDs behavior are determined from forward bias. The current density-voltage characteristics in logarithmic scale for PETMWCNTs/MEH-PPV/Al structures are shown in Figure 4. Indeed, the structure elaborated on the MWCNTs elaborated by spin coating shows a single linear region. The slope value is one, indicating an Ohmic conduction. Therefore, we cannot use the PET-MWCNTs electrode prepared by spin coating as anode. On the other hand, for the structure using PET-MWCNTs anode elaborated through membrane, the curve shows the presence of two regions. We note a transition from a linear to a quadratic variation. In the first 


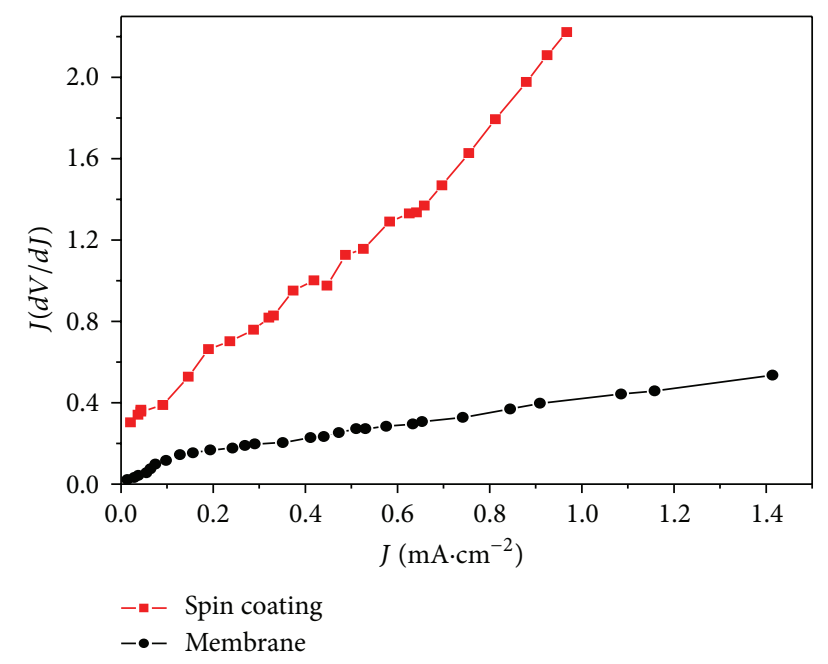

FIgURE 5: $J-J(d V / d J)$ characteristic of PET-MWCNTs/MEH-PPV/ $\mathrm{Al}$ structure.

region $(V<0.65 \mathrm{~V})$, conduction is due to the thermally generated intrinsic charge carriers and the slope value equal to 1 indicates an Ohmic conduction. The second region with a slope value equal to 2 indicates that the current is limited by space charge (SCLC) with a single level traps.

3.3.2. Calculation of Series Resistance by J-J (dV/dJ) Method. $J-V$ characteristic curvature (Figure 4) for forward bias and sufficiently large tension is caused by the presence of series resistance $R_{s}$ [30]. The series resistance $R_{s}$ and the ideality factor $n$ are determined from Figure 5 using a method developed in [31].

At the Schottky contact formed in the semiconductor/metal interface, the current is supposed due to thermoionic emission. By adopting this theory, the current is expressed as

$$
J=J_{s}\left(\exp \left(\frac{q V}{n k T}\right)-1\right)
$$

The following equation presents voltage versus current:

$$
V=\frac{n k T}{q} \ln \left(\frac{J}{J_{s}}+1\right)+J R_{s} .
$$

Taking into account the voltage expression, the voltage derivative with respect to current is given by

$$
\frac{d V}{d J}=\frac{n k T}{q} \frac{1}{J / J_{s}+1} \frac{1}{J_{s}}+R_{s} .
$$

For large values, the total current is greater than the saturation current $\left(J \gg J_{s}\right)$. So $(\mathrm{V} .3)$ leads to

$$
J \frac{d V}{d J}=I R_{s}+\frac{n k T}{q}
$$

With these assumptions, $J(d V / d J)$ depends linearly on $J$. Therefore, the series resistance can be easily obtained from the slope of the region corresponding to the high current by a linear fit. $J(d V / d J)-J$ characteristic is shown in Figure 5. We obtained the following parameters: for the structure prepared on the MWCNTs elaborated by spin coating, the series resistance $R_{s}=312 \Omega$ and ideality factor $n=3.8$. For the structure prepared on the MWCNTs elaborated through membrane, the series resistance $R_{s}=1392 \Omega$ and ideality factor $n=3.8$.

For the structure prepared on the MWCNTs elaborated by spin coating, the series resistance is approximately in a high order. This value is attributed to the anode morphology, the contact resistance and the nature of the PETMWCNTs/MEH-PPV interface. For the structure fabricated on the MWCNTs elaborated through membrane, the series resistance is approximately in a middle order. This value is allocated to (i) more surface heterojunction due to the interpenetration of MEH-PPV and MWCNTs, and (ii) the charge transporting paths are relatively long [32]. In addition, the structure was prepared in air and left in ambient conditions. Therefore, the series resistance value can be affected by many factors, such as reduced mobility and the changes in the contact barrier located at the space charge regions $[33,34]$. The ideality factor $n$ is an important parameter in the specification of the electrical behavior of the diodes which is assigned to the voltage drop in the interfacial layer [35]. This is probably due to the charge overcrowding and the high probability of electrons and holes recombination in the depletion region [36].

3.4. Overview. Our results show that the anode/organic interface plays a crucial role to determine the devices performance. Holes injection process at PET-MWCNTs/MEHPPV interface can be considered as one of the main factors that control the electrical characteristics and performance of the device. It has been shown that holes injection depends on several factors such as the height energy barrier at the anode/organic interface. This energy is determined by the difference between the anode work function and the HOMO level of the organic layer according to the energy levels of the diagram given in Figure 1. After the introduction of the PET-MWCNTs electrode interface, the decrease of holes injection barrier height justifies the improvement of current-voltage characteristics $J(V)$ observed in the modified structure. Therefore, we obtain better diode performance. It is also interesting to note that the improvement of electrical properties highlights the lack of dipoles that can be formed at the anode/organic interface and reduces the diode efficiency [37]. The injection barrier height is not the only factor that controls the holes injection. Indeed, the variation of the anode surface morphology can be also considered as an important factor: a rougher interface can facilitate the charge carriers exchange. Therefore, we can recapitulate that the nature of the surface morphology dependent on MWCNTs film deposits method. It is directly correlated with the decrease of the barrier height. The improvement of the interface electrical parameters using a PET-MWCNTs electrode, elaborated through a membrane, is attributed to the good surface morphology and the contact resistance. In fact, the contact resistance is a significant factor contributing 
to the decrease of the series resistance. This is correlated with the reduction of the hole barrier height.

\section{Conclusion}

The AFM analysis made on CNTs deposited by both methods allowed us to conclude that adopting the second deposition procedure we obtain an oriented and homogeneously distributed CNTs network. The measurement results of the resistivity and the optical transmission show that these electrodes can be used as anodes in flexible optoelectronic devices. Using the $J-J(d V / d J)$ method, we calculated the ideality factor and the series resistance. The results indicate that the holes injection process at the PET-MWCNT/MEHPPV interface is effective. The origin of these improvements is mainly explained by the band structure alignment leading to reduction of the height barrier.

\section{Conflict of Interests}

The authors declare that there is no conflict of interests regarding the publication of this paper.

\section{References}

[1] C. M. Niu, "Carbon nanotube transparent conducting films," MRS Bulletin, vol. 36, no. 10, pp. 766-773, 2011.

[2] T. P. Tyler, R. E. Brock, H. J. Karmel, T. J. Marks, and M. C. Hersam, "Electronically monodisperse single-walled carbon nanotube thin films as transparent conducting anodes in organic photovoltaic devices," Advanced Energy Materials, vol. 1, no. 5, pp. 785-791, 2011.

[3] D. R. Kauffman, D. C. Sorescu, D. P. Schofield, B. L. Allen, K. D. Jordan, and A. Star, "Understanding the sensor response of metal-decorated carbon nanotubes," Nano Letters, vol. 10, no. 3, pp. 958-963, 2010.

[4] S. De and J. N. Coleman, "The effects of percolation in nanostructured transparent conductors," MRS Bulletin, vol. 36, no. 10, pp. 774-781, 2011.

[5] A. C. Tolcin, "Mineral commodity summaries," Indium, vol. 1, pp. 74-75, 2013.

[6] H. Karimi-Maleh, F. Tahernejad-Javazmi, N. Atar, M. L. Yola, V. K. Gupta, and A. A. Ensafi, "A novel DNA biosensor based on a pencil graphite electrode modified with polypyrrole/functionalized multiwalled carbon nanotubes for determination of 6-mercaptopurine anticancer drug," Industrial \& Engineering Chemistry Research, vol. 54, no. 14, pp. 3634-3639, 2015.

[7] N. Atar, T. Eren, M. L. Yola, H. Karimi-Maleh, and B. Demirdögen, "Magnetic iron oxide and iron oxide@gold nanoparticle anchored nitrogen and sulfur-functionalized reduced graphene oxide electrocatalyst for methanol oxidation," RSC Advances, vol. 5, no. 33, pp. 26402-26409, 2015.

[8] D. Zhang, K. Ryu, X. Liu et al., “Transparent, conductive, and flexible carbon nanotube films and their application in organic light-emitting diodes," Nano Letters, vol. 6, no. 9, pp. 1880-1886, 2006.

[9] J. Li, L. Hu, L. Wang, Y. Zhou, G. Grüner, and T. J. Marks, "Organic light-emitting diodes having carbon nanotube anodes," Nano Letters, vol. 6, no. 11, pp. 2472-2477, 2006.
[10] M. Bansal, R. Srivastava, C. Lal, M. N. Kamalasanan, and L. S. Tanwar, "Carbon nanotube-based organic light emitting diodes," Nanoscale, vol. 1, no. 3, pp. 317-330, 2009.

[11] S. Shi and S. R. P. Silva, "High luminance organic lightemitting diodes with efficient multi-walled carbon nanotube hole injectors," Carbon, vol. 50, no. 11, pp. 4163-4170, 2012.

[12] A. R. Inigo, J. M. Underwood, and S. R. P. Silva, "Carbon nanotube modified electrodes for enhanced brightness in organic light emitting devices," Carbon, vol. 49, no. 13, pp. 4211-4217, 2011.

[13] N. A. Nismy, K. D. G. I. Jayawardena, A. A. D. T. Adikaari, and S. R. P. Silva, "Photoluminescence quenching in carbon nanotubepolymer/fullerene films: carbon nanotubes as exciton dissociation centres in organic photovoltaics," Advanced Materials, vol. 23, no. 33, pp. 3796-3800, 2011.

[14] N. Senthilkumar, H.-S. Kang, D.-W. Park, and Y. Choe, "Improving efficiency of organic photovoltaic cells using PEDOT:PSS and MWCNT nanocomposites as a hole conducting layer," Journal of Macromolecular Science, Part A: Pure and Applied Chemistry, vol. 47, no. 5, pp. 484-490, 2010.

[15] C. D. Williams, R. O. Robles, M. Zhang, S. Li, R. H. Baughman, and A. A. Zakhidov, "Multiwalled carbon nanotube sheets as transparent electrodes in high brightness organic light-emitting diodes," Applied Physics Letters, vol. 93, no. 18, Article ID 183506, 2008.

[16] W. Aloui, A. Ltaief, and A. Bouazizi, "Transparent and conductive multi walled carbon nanotubes flexible electrodes for optoelectronic applications," Superlattices and Microstructures, vol. 64, pp. 581-589, 2013.

[17] K. B. Hartman and L. J. Wilson, "Carbon nanostructures as a new high-performance platform for MR molecular imaging," in Bio-Applications of Nanoparticles, vol. 620 of Advances in Experimental Medicine and Biology, pp. 74-84, Springer, Berlin, Germany, 2007.

[18] G. Xiao, Y. Tao, J. Lu, and Z. Zhang, "Highly conductive and transparent carbon nanotube composite thin films deposited on polyethylene terephthalate solution dipping," Thin Solid Films, vol. 518, no. 10, pp. 2822-2824, 2010.

[19] A. A. Kuznetzov, S. B. Lee, M. Zhang, R. H. Baughman, and A. A. Zakhidov, "Electron field emission from transparent multiwalled carbon nanotube sheets for inverted field emission displays," Carbon, vol. 48, no. 1, pp. 41-46, 2010.

[20] Y. Zhou, L. Hu, and G. Grüner, "A method of printing carbon nanotube thin films," Applied Physics Letters, vol. 88, no. 12, pp. 123109-123112, 2006.

[21] W.-Y. Ko, J.-W. Su, C.-H. Guo, S.-J. Fu, C.-Y. Hsu, and K.-J. Lin, "Highly conductive, transparent flexible films based on open rings of multi-walled carbon nanotubes," Thin Solid Films, vol. 519, no. 22, pp. 7717-7722, 2011.

[22] H.-Z. Geng, K. K. Ki, P. S. Kang, S. L. Young, Y. Chang, and H. L. Young, "Effect of acid treatment on carbon nanotube-based flexible transparent conducting films," Journal of the American Chemical Society, vol. 129, no. 25, pp. 7758-7759, 2007.

[23] M. J. de Andrade, M. D. Lima, V. Skákalová, C. P. Bergmann, and S. Roth, "Electrical properties of transparent carbon nanotube networks prepared through different techniques," Physica Status Solidi (RRL)_Rapid Research Letters, vol. 1, no. 5, pp. 178-180, 2007.

[24] M. J. Kim, D. W. Shin, J.-Y. Kim, S. H. Park, I. T. Han, and J. B. Yoo, "The production of a flexible electroluminescent device on polyethylene terephthalate films using transparent conducting 
carbon nanotube electrode," Carbon, vol. 47, no. 15, pp. 34613465, 2009.

[25] Y. Jia, X. Li, P. Li et al., "Strong, conductive carbon nanotube fibers as efficient hole collectors," Nanoscale Research Letters, vol. 7, article 137, 2012.

[26] J. Cui, A. Wang, N. L. Edleman et al., "Indium tin oxide alternatives-high work function transparent conducting oxides as anodes for organic light-emitting diodes," Advanced Materials, vol. 13, no. 19, pp. 1476-1480, 2001.

[27] Y. Wang, C.-A. Di, Y. Liu et al., "Optimizing single-walled carbon nanotube films for applications in electroluminescent devices," Advanced Materials, vol. 20, no. 23, pp. 4442-4449, 2008.

[28] M. R. S. Castro and H. K. Schmidt, "Preparation and characterization of low- and high-adherent transparent multi-walled carbon nanotube thin films," Materials Chemistry and Physics, vol. 111, no. 2-3, pp. 317-321, 2008.

[29] S. Hameed, P. Predeep, and M. R. Baiju, "Polymer light emitting diodes-a review on materials and techniques," Reviews on Advanced Materials Science, vol. 26, no. 1-2, pp. 30-42, 2010.

[30] S. Aydogan, M. Saglam, and A. Turut, "Some electrical properties of polyaniline/p-Si/Al structure at $300 \mathrm{~K}$ and $77 \mathrm{~K}$ temperatures," Microelectronic Engineering, vol. 85, no. 2, pp. 278-283, 2008.

[31] Y. Shen, K. Li, N. Majumdar, J. C. Campbell, and M. C. Gupta, "Bulk and contact resistance in P3HT:PCBM heterojunction solar cells," Solar Energy Materials and Solar Cells, vol. 95, no. 8, pp. 2314-2317, 2011.

[32] V. S. Balderrama, M. Estrada, A. Cerdeira et al., "Influence of P3HT:PCBM blend preparation on the active layer morphology and cell degradation," Microelectronics Reliability, vol. 51, no. 3, pp. 597-601, 2011.

[33] M. Glatthaar, N. Mingirulli, B. Zimmermann et al., "Impedance spectroscopy on organic bulk-heterojunction solar cells," Physica Status Solidi (A), vol. 202, no. 11, pp. R125-R127, 2005.

[34] M. J. Deen and M. H. Kazemeini, "Photosensitive polymer thinfilm FETs based on poly(3-octylthiophene)," Proceedings of the IEEE, vol. 93, no. 7, pp. 1312-1320, 2005.

[35] M. H. Suhail, I. M. Ibrahim, Z. Ahmad, S. M. Abdullah, and K. Sulaiman, "ITO/PEDOT: PSS/MEH: PPV/Alq3/LiF/Au as a schottky diode," International Journal of Application or Innovation in Engineering \& Management, vol. 2, no. 1, pp. 130136, 2013.

[36] M. M. El-Nahass, A. M. Farid, A. A. M. Farag, and H. A. M. Ali, "Carrier transport mechanisms and photovoltaic characteristics of p- $\mathrm{H}_{2} \mathrm{Pc} / \mathrm{n}$-Si heterojunctions," Vacuum, vol. 81, no. 1, pp. 8-12, 2006.

[37] B. Kouskoussa, M. Morsli, K. Benchouk et al., "On the improvement of the anode/organic material interface in organic solar cells by the presence of an ultra-thin gold layer," Physica Status Solidi (A), vol. 206, no. 2, pp. 311-315, 2009. 

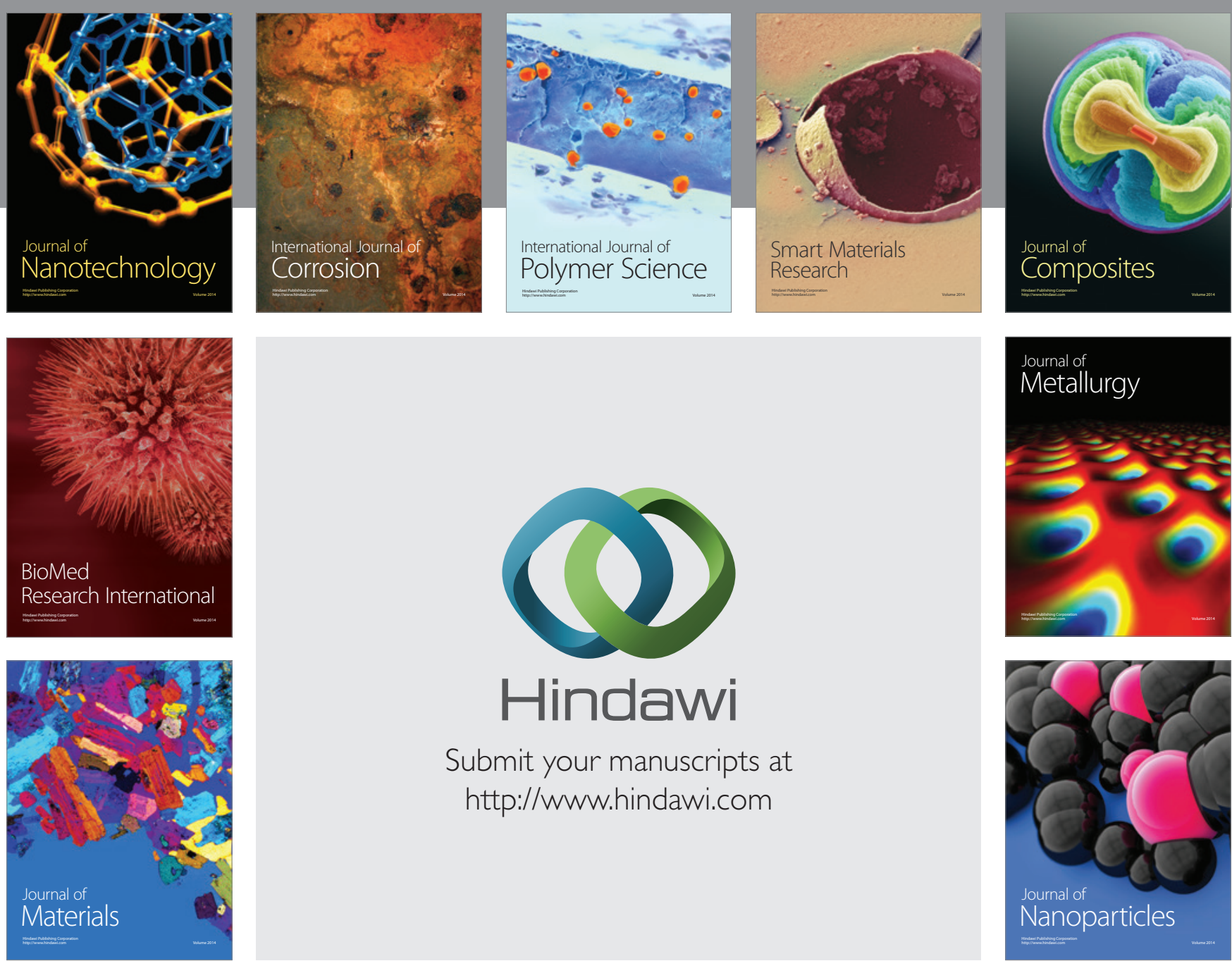

\section{Hindawi}

Submit your manuscripts at

http://www.hindawi.com

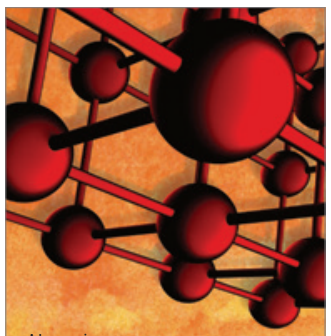

Materials Science and Engineering
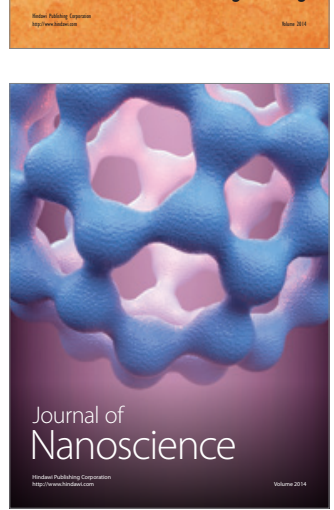
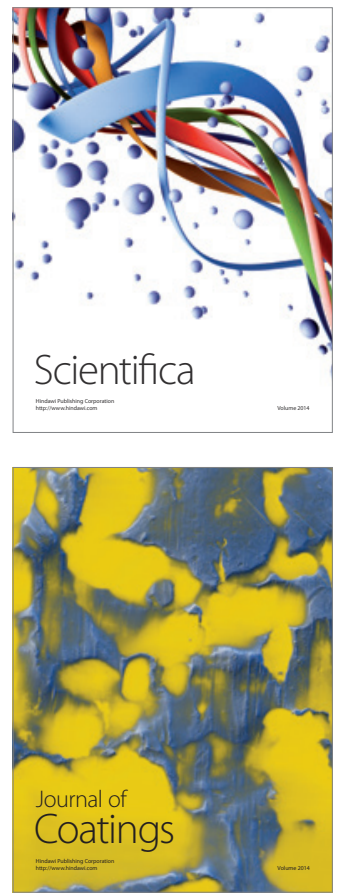
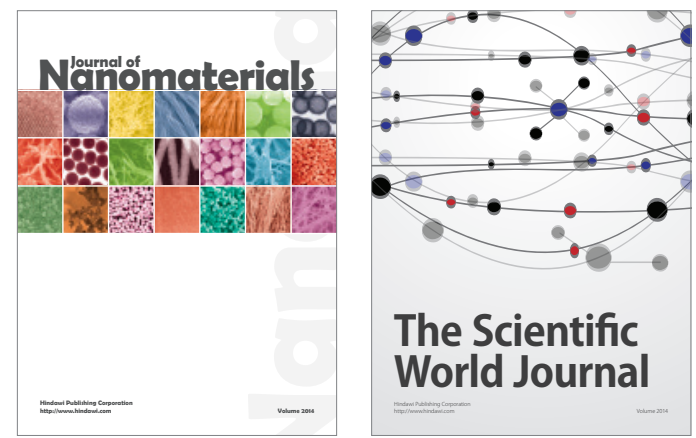

The Scientific World Journal
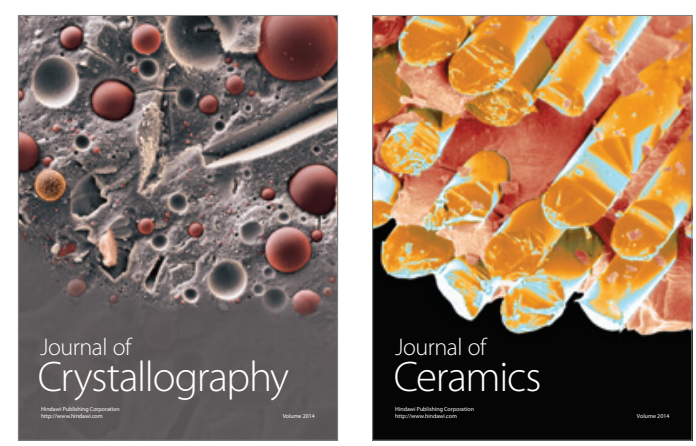
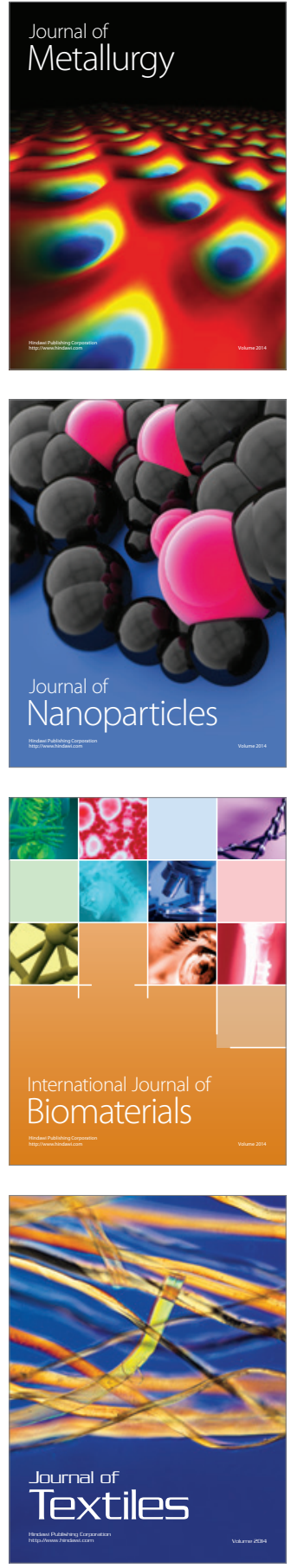